\title{
Differential FSH Glycosylation Modulates FSHR Oligomerization and Subsequent cAMP Signaling
}

\author{
Uchechukwu T. Agwuegbo ${ }^{1}$, Emily Colley ${ }^{2}$, Anthony P. Albert ${ }^{3}$, Viktor Y. Butnev ${ }^{4}$, \\ George R. Bousfield ${ }^{4}$ and Kim C. Jonas ${ }^{1 *}$
}

1 School of Life Course and Population Sciences, Department of Women and Children's Health, Guy's Campus, King's College London, London, United Kingdom, ${ }^{2}$ Institute of Reproductive and Developmental Biology, Imperial College London, London, United Kingdom, ${ }^{3}$ Vascular Biology Research Centre, Molecular \& Clinical Science Research Centre, St George's University of London, London, United Kingdom, ${ }^{4}$ Department of Biological Sciences, Wichita State University, Wichita, KS, United States

OPEN ACCESS

Edited by:

Livio Casarini,

University of Modena and Reggio

Emilia, Italy

Reviewed by:

Mohammed Akli AYOUB,

United Arab Emirates University,

United Arab Emirates

Francesco Poti,

University of Parma, Italy

*Correspondence:

Kim C. Jonas

kim.jonas@kcl.ac.uk

Specialty section:

This article was submitted to

Reproduction,

a section of the journal

Frontiers in Endocrinology

Received: 27 August 2021 Accepted: 02 November 2021 Published: 03 December 2021

Citation:

Agwuegbo UT, Colley E,

Albert AP, Butnev VY,

Bousfield GR and Jonas KC (2021)

Differential FSH Glycosylation Modulates FSHR Oligomerization and Subsequent CAMP Signaling.

Front. Endocrinol. 12:765727. doi: 10.3389/fendo.2021.765727
Follicle-stimulating hormone (FSH) and its target G protein-coupled receptor (FSHR) are essential for reproduction. Recent studies have established that the hypo-glycosylated pituitary FSH glycoform (FSH21/18), is more bioactive in vitro and in vivo than the fullyglycosylated variant (FSH24). FSH21/18 predominates in women of reproductive prime and FSH24 in peri-post-menopausal women, suggesting distinct functional roles of these FSH glycoforms. The aim of this study was to determine if differential FSH glycosylation modulated FSHR oligomerization and resulting impact on cAMP signaling. Using a modified super-resolution imaging technique (PD-PALM) to assess FSHR complexes in HEK293 cells expressing FSHR, we observed time and concentration-dependent modulation of FSHR oligomerization by FSH glycoforms. High eFSH and FSH21/18 concentrations rapidly dissociated FSHR oligomers into monomers, whereas FSH24 displayed slower kinetics. The FSHR $\beta$-arrestin biased agonist, truncated eLH $\beta(\Delta 121$ 149) combined with asparagine56-deglycosylated eLH $\alpha$ (dg-eLHt), increased FSHR homomerization. In contrast, low FSH21/18 and FSH24 concentrations promoted FSHR association into oligomers. Dissociation of FSHR oligomers correlated with time points where higher CAMP production was observed. Taken together, these data suggest that FSH glycosylation may modulate the kinetics and amplitude of cAMP production, in part, by forming distinct FSHR complexes, highlighting potential avenues for novel therapeutic targeting of the FSHR to improve IVF outcomes.

Keywords: follicle-stimulating hormone receptor, follicle-stimulating hormone, gonadotropic hormones, G proteincoupled receptors (GPCR), oligomers, oligomerization

\section{INTRODUCTION}

The actions of follicle-stimulating hormone (FSH) and its receptor (FSHR) are essential for reproduction (1-5). With critical roles in follicle maturation, recruitment, and dominant follicle selection, FSH/FSHR are pivotal for granulosa cell (GC) proliferation and estradiol production $(6,7)$. Consequently, FSHR is a key therapeutic target of assisted reproductive technologies (ART), where supraphysiological concentrations of recombinant and urinary FSH preparations are utilized 
during the ovarian stimulation phase of in vitro fertilization (IVF) to facilitate the recruitment and maturation of multiple antral follicles (8-10). Despite technological advances in IVF, there has been little change in the success rates (11), which are highest in women $<35$ and decline thereafter, highlighting the need for novel therapeutic FSH/FSHR targeting strategies to advance IVF success rates.

FSH is a complex heterodimeric glycoprotein hormone, comprised of an alpha subunit, that is common to other glycoprotein hormone family members such as thyroidstimulating hormone (TSH), luteinizing hormone (LH) and human chorionic gonadotropin hormone (hCG), along with a hormone specific beta subunit (FSH $\beta$ ). FSH $\beta$ differs in amino acid sequence and glycosylation pattern to other glycoprotein hormone beta subunits, conferring biological specificity and selectivity of FSH to FSHR (12). FSH possesses 4 Asn-linked glycosylation sites, with two sites on the alpha subunit (Asn52 and Asn78) and two sites on the beta subunit (Asn7 and Asn24). Naturally occurring differences in the macro-glycosylation pattern of FSH have been previously reported in human pituitary extracts (13), with modification of the FSH $\beta$ subunit glycosylation pattern resulting in the identification of three FSH glycoforms; partially glycosylated FSH (FSH21/18, as purified preparations possess both variants) with a single glycosylated site at either Asn7 (FSH21) or Asn24 (FSH18) and fully glycosylated FSH (FSH24) with both FSH $\beta$ subunit Asn residues glycosylated. Interestingly, age-dependent differences in pituitary expression levels of FSH21/18 and FSH24 have been reported. FSH21/18 has been shown to be predominant in pituitary extracts from women in their 20's, decreasing thereafter, with a concomitant increase in $\mathrm{FSH} 24$ expression, resulting in $\mathrm{FSH} 24$ predominating in menopausal-aged women (13). Functional analysis has shown that this difference in glycosylation pattern results in modulation of binding to the FSHR (14) and the amplitude of the canonical FSHR signaling pathway, the Gos/cAMP/PKA signaling, with FSH21/18 displaying faster binding kinetics and more potent activation of Gos signaling (15-17). Recent studies suggest that FSH glycoforms may display distinct signaling profiles, or signal bias (18) in activation of $\operatorname{AKT}(19,20), \beta$-arrestin (17) and calcium signaling $(17,21)$. Important functional differences have also been observed, with differences in ovarian and testicular gene expression in mice injected with either FSH21/18 or FSH24 (19). However, how these differences in the signaling properties of FSH21/18 and FSH24 are interpreted by FSHR remain unknown.

FSHR is a Class A G protein-coupled receptor (GPCR). An increasingly important way that GPCRs have been shown to regulate ligand specificity and signal amplitude is via association and formation of dimers/oligomers (22-25). FSHR has been demonstrated to self-associate and homomerize (26-30), which is thought to underpin the inherent negative cooperativity displayed by FSHR (26). The FSHR has also been shown to heteromerize with the LHR (30-32) and membrane-bound estrogen receptor (GPER) (33), resulting in modulation of signal selectivity $(31,32)$. However, the functional role of FSHR homomerization remains to be demonstrated, and how different FSH ligands may impact FSHR homomerization to mediate their differences in signal specificity and selectivity remains unknown.

With advances in single molecule imaging technology, we are now in the position to investigate the molecular mechanisms underpinning how FSH glycoforms specify the differences observed in the kinetics and amplitude of cAMP signaling, with single molecule precision and at physiological levels of receptor density. Using a combination of the single molecule imaging technique, photoactivated dye-localization microscopy (PD-PALM) $(34,35)$, and differential FSH glycosylation variants- FSH21/18, FSH24, a potent FSHR stimulator- equine FSH (eFSH) and a FSHR $\beta$-arrestin biased agonist with diminished ability to activate cAMP - truncated eLH $\beta(\Delta 121$ 149) combined with asparagine56-deglycosylated eLH $\alpha$, designated dg-eLHt (36), we have determined that FSH glycoforms differentially modulate FSHR oligomerization in both a temporal and concentration-dependent manner. These differences observed in FSHR oligomerization correlated with temporal and magnitude differences observed in cAMP production and cre-luciferase activity. These data suggest a novel mechanism by which different FSH ligands may modulate the magnitude of cAMP signal through differential regulation of FSHR oligomerization.

\section{MATERIAL AND METHODS}

\section{Materials}

Purified pituitary FSH21/18 and FSH24 (17), equine FSH (eFSH) and dg-eLHt (37) were kindly supplied by Professor George Bousfield, Wichita State University, Wichita, KS, USA. CAGE 552 fluorophore dye was purchased from Abberior. N-terminally hemagglutinin-tagged FSHR (HA-FSHR) encoded plasmid DNA were constructed as previously described (32). Primary antibody HA.11 was purchased from BioLegend ${ }^{\circledR}$. Plasmid DNA encoding GloSensor ${ }^{\mathrm{TM}}$-20F, cAMP-response element-luciferase reporter gene (cre-luciferase), Renilla luciferase reporter gene $(R$ luciferase), GloSensor ${ }^{\mathrm{TM}}$ reagent stock and Dual-Luciferase ${ }^{\circledR}$ Reporter Assay System were purchased from Promega.

\section{Cell Culture and Transient Transfections}

HEK293 cells were maintained and cultured in 5\% $\mathrm{CO}_{2}$ in air at $37^{\circ} \mathrm{C}$ in Dulbecco's Modified Eagle's Medium (DMEM-6429, Sigma-Aldrich) supplemented with $10 \%$ Fetal Bovine Serum (FBS-F9665, Sigma-Aldrich), and 1\% Antibiotic-Antimycotic (15240062, ThermoFisher). For PD-PALM experiments, cells were transiently transfected with $3 \mu \mathrm{g}$ HA-FSHR, and then replated 24 hours later onto 8-well 1.5 glass-bottomed chamber slides. For cAMP GloSensor ${ }^{\mathrm{TM}}$ and cre-luciferase activity assays, HEK293 cells were co-transiently co-transfected with $3 \mu \mathrm{g}$ of HA-FSHR and either $1 \mu \mathrm{g}$ GloSensor ${ }^{\mathrm{TM}}$-20F DNA plasmid or $800 \mathrm{ng}$ cre-luciferase and $150 \mathrm{ng} R$-luciferase DNA plasmids, and cells re-plated the following day onto white clear-bottomed 96well plates $(50,000$ cells/well). All transient transfections were carried out in tissue culture-treated 6-well plates $(600,000$ cells/well) 
using Lipofectamine $2000^{\circledR}$ (Invitrogen) as per manufacturer's instructions. Cells were assayed 48 hours post-transfection.

\section{PD-PALM}

To assess FSHR monomer, dimer and oligomer populations at the plasma membrane, PD-PALM experiments were performed as previously described $(32,34,35)$. Briefly, HEK293 cells expressing HA-FSHR were pre-incubated for 30 minutes with CAGE 552-labeblled HA.11 antibody at $37^{\circ} \mathrm{C}$, protected from light. HA.11 primary antibodies were labeled with CAGE 552 photoswitchable dyes at a 1:1 ratio, as previously described (34, 35) and following manufacturer's protocols (Abberior). At the end of the pre-incubation, antibodies were removed, and cells were treated with 0 (control), or either 30 or $1 \mathrm{ng} / \mathrm{ml} \mathrm{of} \mathrm{eFSH,}$ FSH21/18, FSH24 or dg-eLHt for 0, 2-, 5- or 15 minutes. Cells were washed with PBS and fixed for 30 minutes with $0.2 \%$ glutaraldehyde in 4\% PFA at room temperature. Following fixation, cells were subsequently washed, stored and imaged in PBS using an inverted Zeiss Elyra PS.1 microscope with a 100x 1.45 NA objective lens in TIRF-mode at a rate of 10 frames/ second over a total of 31,500 frames.

\section{Localization Analysis}

Individual FSHR molecules were resolved as previously described (32, 34, 35). To summarize, cropped non-overlapping 5 x $5 \mu \mathrm{m}$ areas, within cell boundaries, of fluorescent intensity image frames were analyzed using the QuickPALM Fiji plug-in, generating $x-y$ coordinates of each FSHR molecule. The range of FSHR density basally observed at the cell surface was $10-80 \mathrm{FSHR} / \mu \mathrm{m}^{2}$ across all experiments. For standardization of data analysis and to eliminate receptor density as a variable factor, cells with FSHR expression levels of $\sim 10-40 \mathrm{FSHR} / \mu^{2}$ were selected for analysis, as this was the physiological receptor density range previously reported for the FSHR (30) and other native GPCRs (38). To prevent the overestimation of FSHR association, coordinates localized within $15 \mathrm{~nm}$ of 15 consecutive frames were filtered using an add-on algorithm JAVA program. To determine the number of associated FSHRs and type of associated forms, from the localized and filtered $\mathrm{x}-\mathrm{y}$ coordinates, a PD-interpreter JAVA program was employed to perform Getis-Franklin neighborhood analysis with a search radius of $50 \mathrm{~nm}$. Reconstructed heat maps representing the different numbers of associated FSHRs were produced as a result.

\section{GloSensor $^{\mathrm{TM}}$ cAMP Assay}

Post-transfection, cells were pre-equilibrated for 2 hours at $37^{\circ} \mathrm{C}$ in $88 \% \mathrm{CO}_{2}$-independent media (18045, Gibco) supplemented with $10 \%$ FBS and $2 \%$ GloSensor ${ }^{\text {TM }}$ cAMP reagent stock, as per manufacturer's instructions. Following this, cells were treated for 30 minutes at $37^{\circ} \mathrm{C}$ with $0-100 \mathrm{ng} / \mathrm{ml}$ of eFSH, FSH21/18, FSH24 or dg-eLHt. Real-time cAMP florescence was measured using a multi-mode plate reader (PHERAstar ${ }^{\circledR}$ FS, BMG Labtech) using the parameter of 100 flashes per well, with a cycle time of 36 seconds.

\section{Cre-Luciferase Assay}

Post-transfection, cells were stimulated in serum-free DMEM supplemented and treated with 0-100 ng/ml eFSH, FSH21/18,
$\mathrm{FSH} 24$ or dg-eLHt for 4-6 hours at $37^{\circ} \mathrm{C}$. As an early-response gene, we expected this length of treatment to be sufficient for rapid gene expression induction in concordance with previous work (15). At the end of the incubation period, cells were lysed and treated with the Dual-Luciferase ${ }^{\circledR}$ Reporter Assay System, as per manufacturer's instructions. Lysate preps were measured for cre-luciferase and $R$-luciferase (for internal transfection control) luminescence levels using a multi-mode plate reader (PHERAstar ${ }^{\circledR}$ FS, BMG Labtech).

\section{Statistical Analysis}

For PD-PALM studies, to compare the effect of FSHR ligands on the percentage of total FSHR homomers, ordinary one-way ANOVA, followed by Tukey's multiple comparisons test were conducted. To compare the effect of different FSHR ligands on the percentage of FSHR homomer subtypes, we performed multiple unpaired t-tests followed by Holm-Sídák's multiple comparisons. For each experiment a total of 3 individual sections/well were imaged containing 3-4 cells. For each section typically 15 ROIs, within cell borders, were analyzed as previously described (34). For GloSensor ${ }^{\mathrm{TM}}$ assays, a baseline read of each well was performed for 10 read cycles prior to FSHR ligand treatment. The average baseline value of each cell was subtracted from its respective FSHR ligand-treatment, from the same wells. The mean cAMP response was plotted over 30 minutes and second order smoothened graph with 10 neighbors was performed. The area under the curve (AUC) measurements at 2-, 5- and 15-minutes were determined by measuring the total area from the number of peaks. All GloSensor $^{\text {TM }}$ data were represented as fold change/basal. Comparisons of the AUC between FSHR ligands were carried out using one-way ANOVA, followed by Tukey's multiple comparisons test. Cre-luciferase luminescence readings were normalized to $R$-luciferase luminescence readings from the same well, to control for transfection efficiency. All data were represented as fold change/basal. Analysis of concentrationresponse curve were made using two-way ANOVA, followed by Dunnett's multiple comparisons test. Comparisons between FSHR ligands at specific concentrations were performed using two-way ANOVA, followed by Dunnett's multiple comparisons test. For PD-PALM, a minimum of 3 independent experiments were performed, for GloSensor ${ }^{\mathrm{TM}}$ and cre-luciferase assays, a minimum of 3 independent experiments in triplicate were conducted. All data presented represent the mean \pm SEM. All statistical evaluations were performed using GraphPad Prism V9, and significance was determined as a probability value of $p<0.05$.

\section{RESULTS}

\section{FSH Ligands Differentially Modulate FSHR Monomer and Homomer Complex Formation in a Temporal and Concentration-Dependent Manner}

To determine the effects of differentially glycosylated FSHR ligands on cell surface FSHR oligomerization, we utilized the 
previously described single molecule imaging technique, PDPALM, which afforded imaging of individual FSHR molecules to $<10 \mathrm{~nm}$ resolution (34). Two concentrations of FSH glycoforms were utilized, based on previous reports showing differential cAMP production evoked by FSH21/18 and FSH24, with $\sim 50 \%$ of maximal cAMP production at $30 \mathrm{ng} / \mathrm{ml}$ in the concentration ranges assessed, and low-level cAMP production at $1 \mathrm{ng} / \mathrm{ml}(15)$.

HEK293 cells transiently expressing HA-FSHR were treated \pm $30 \mathrm{ng} / \mathrm{ml}$ of FSH21/18 and FSH24 for 2-, 5- and 15 minutes. As a positive control, cells were also stimulated with the potent FSHR activator, eFSH, which is a naturally occurring analog of hypoglycosylated FSH21/18. Conversely, as a negative control for cAMP activation, we utilized the FSHR $\beta$-arrestin biased agonist, dg-eLHt, which has been previously shown to display minimal cAMP production, with biased activation of $\beta$-arrestin $(36,37)$. Representative images (top panels) and heat maps depicting the number of associated molecules (bottom panels) were generated. Analysis of the basal number of associated FSHR showed that $30.2 \pm 1.8 \%$ of FSHR were associated as dimers and oligomers (Figure 1), with $\sim 70 \%$ as FSHR monomers. Analysis of the basal composition of associated cell surface FSHR showed $15.5 \pm 0.8 \%$ resided as dimers and $5.5 \pm 0.5 \%$ as trimers (Figure 1), suggesting that basally the majority of FSHR reside as lowerorder homomers and monomers. Acute 2-minute treatment of HEK293 cells expressing FSHR with either eFSH or FSH21/18 significantly decreased the overall percentage of associated FSHR, with $20.0 \pm 1.3 \%$ and $17.5 \pm 1.6 \%$ associated as dimers and oligomers, respectively (Figure 1Aii). A decrease was observed in almost all FSHR homomeric subtypes (dimers, trimers, pentamers and 6-8 oligomers) (Figure 1Aiii). In contrast, treatment with $\mathrm{FSH} 24$ had no effect on the total percentage of associated FSHR, however modulation in the type of FSHR homomeric complexes was observed with a modest increase in $\geq 9$ complexes and a decrease in dimers. Surprisingly, 2-minute treatment with dg-eLHt showed a trend for increasing FSHR association with $38.7 \pm 3.8 \%$ of FSHR molecules associated (Figure 1Aii), with $15.9 \pm 2.9 \%$ FSHR as trimers (Figure 1Aiii).

A 5-minute stimulation with eFSH treatment showed the percentage of FSHR associations to resemble basal (Figure 1Bi), suggesting a rapid re-organization of FSHR monomers into FSHR homomers. The documented fast binding kinetics of eFSH may explain this (17). FSH21/18, however, maintained a sustained reduction in FSHR homomers (Figure 1Bi). Interestingly, 5-minute treatment with $\mathrm{FSH} 24$ resulted in FSHR dissociation (Figure 1Bi), with a decrease in dimeric and trimeric FSHR homomers $(p<0.001)$ (Figure 1Bii). A 5minute dg-eLHt treatment sustained the increase in FSHR association observed at 2 minute-treatment (Figure 1Bi), suggesting that different FSH ligands have distinct effects on FSHR oligomerization.

A more chronic 15-minute treatment with either eFSH, FSH21/18 or FSH24 resulted in FSHR total homomeric complex percentages resembling those of basal levels (Figure 1Ci). However, dg-eLHt-treated cells continued to show increased FSHR association $(49.7 \pm 6.4 \%)$ with increases observed in trimers, tetramers to $\geq 9$ complexes (Figure 1Cii), further supporting the proposition that different FSH ligands can differentially modulate FSHR association.

Since FSH concentrations and glycosylation patterns are differentially regulated across the menstrual cycle (39) and have also been shown to change with age (13), we sought to determine the effects of FSH ligand concentration on FSHR association. As previously, HEK293 cells expressing FSHR were treated $\pm \mathrm{eFSH}, \mathrm{FSH} 21 / 18$, FSH24 or dg-eLHt for 2-, 5- or 15minutes, but using $1 \mathrm{ng} / \mathrm{ml}$ of each. Representative reconstructed images of FSHR localizations and heat maps showing associated molecules following 2-minute treatment were generated (Figure 2Ai). Assessment of FSHR association following 2minute treatment with all ligands revealed no significant changes in the total percentage of FSHR homomers (Figure 2Aii), nor the type of FSHR homomeric complexes observed (Figure 2Aiii), suggesting that lower concentrations of FSHR ligands had little effect on FSHR association at this acute time-point.

Similarly, 5-minute treatment with $1 \mathrm{ng} / \mathrm{ml} \mathrm{eFSH,} \mathrm{FSH21/18}$ and dg-eLHt had no effect on FSHR association (Figure 2Bi). Interestingly, FSH24 induced a significant increase in FSHR association, with an increase in the formation of pentamers $(6.1 \pm 2.6 \%)$ (Figure 2Bii), contrasting to the dissociation of FSHR homomers observed with $30 \mathrm{ng} / \mathrm{ml} \mathrm{FSH24}$ shown above. Intriguingly, 15-minute treatment with FSH21/18 also induced FSHR association (Figure 2Ci), with an increase in FSHR tetramers $(9.0 \pm 1.8 \%)$ and $\geq 9$ oligomers $(18.2 \pm 4.1 \%)$ (Figure 2Cii). FSH24-treated cells appeared to show FSHRs return to basal configuration (Figure 2C). Taken together, these data suggest that different FSHR ligands specify distinct re-organization of FSHR monomer, dimer, and oligomer populations in both a concentration- and timedependent manner.

\section{FSHR Ligands Differentially Modulate cAMP Production}

Ligand-dependent modulation of the related luteinizing hormone receptor homomers and LHR/FSHR and FSH/GPER heteromers has been shown to regulate signal amplitude and specificity (32-34) To investigate if the time- and concentrationdependent changes in FSHR monomers and homomers observed at the plasma membrane correlated with modulation in cAMP signals in our cell system, we employed the cAMP GloSensor ${ }^{\mathrm{TM}}$ reporter, which afforded real-time monitoring of cAMP production. HEK293 cells expressing FSHR were treated \pm 0 $100 \mathrm{ng} / \mathrm{ml}$ of eFSH, FSH21/18, FSH24 or dg-eLHt. Full cAMP concentration-response curves showing the AUC and maximal response of cAMP accumulation were recorded (Supplementary Figure S1), and the 30- and $1 \mathrm{ng} / \mathrm{ml}$ data extrapolated for further analysis at the 2-, 5-, and 15-minute time points, for correlation with the time points utilized for PD-PALM experiments. The mean cAMP accumulated over 30 minutes following a $30 \mathrm{ng} / \mathrm{ml}$ treatment with all ligands were plotted (Figure 3Ai). A 2-minute treatment with either eFSH and FSH21/18 induced a significant 
A

(i)
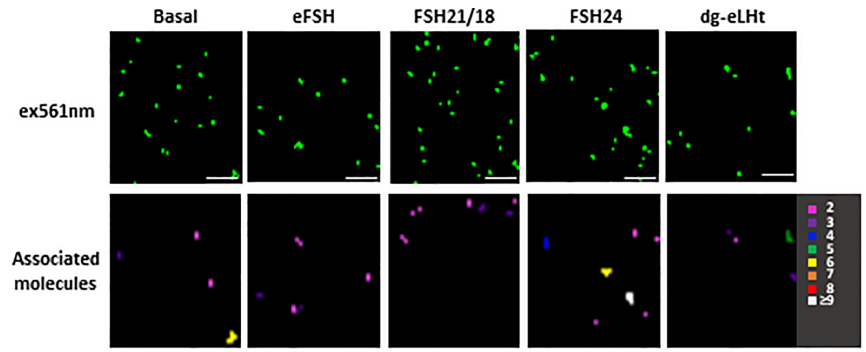

(ii)

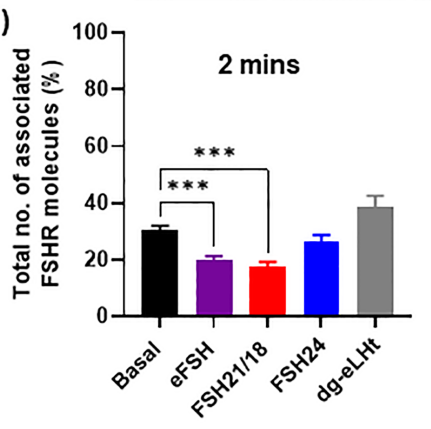

(i)

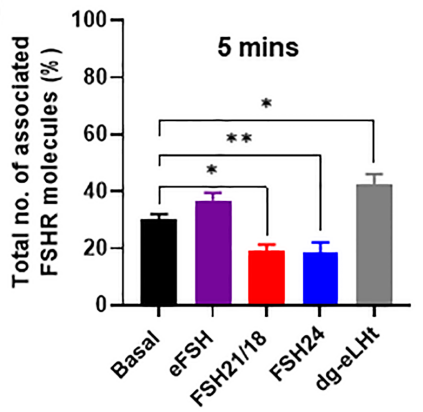

(i)

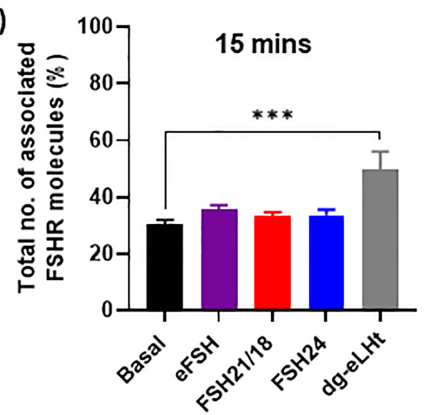

(iii)

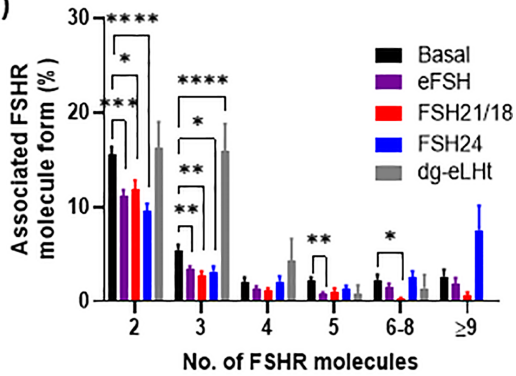

(ii)

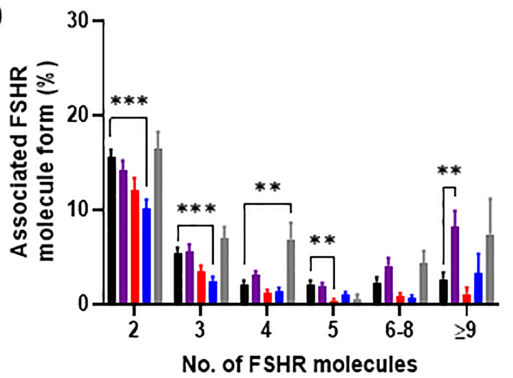

(ii)

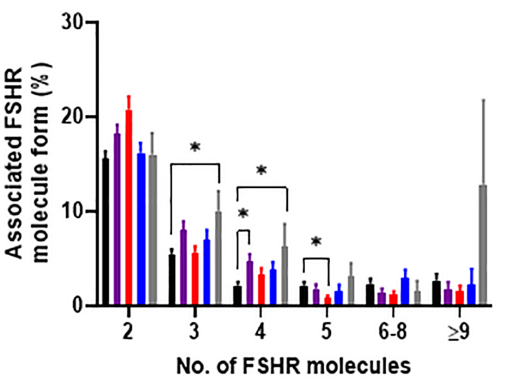

FIGURE 1 | FSH ligands differentially modulate FSHR association. HEK293 cells transiently expressing HA-tagged FSHR were pre-incubated for 30 minutes with CAGE 552-HA antibody and treated with $\pm 30 \mathrm{ng} / \mathrm{ml}$ of eFSH, FSH21/18, FSH24 or dg-eLHt for either (A) 2 minutes, (B) 5 minutes or (C) 15 minutes, fixed for 30 minutes and imaged via PD-PALM. (Ai) Representative $x-y$ coordinate plots of resolved FSHR molecules (upper panel) and reconstructed heat map of FSHR molecules following 2-minute treatment (lower panel). Images are $2 \mu \mathrm{m}^{2}$ from a $5 \mu \mathrm{m}^{2}$ area. Scale bars, $500 \mathrm{~nm}$. (Aii), (Bi) and (Ci) Show the percentage of the total number of associated FSHR molecules; data analyzed using ordinary one-way ANOVA. (Aiii), (Bii) and (Cii) Shows the percentage of associated FSHR molecule form; 2 (dimer), 3 (trimer), 4 (tetramer), 5 (pentamer), 6-8, $\geq 9$, with data analyzed using multiple unpaired t-tests. All data represent mean \pm SEM of 3 independent experiments. ${ }^{*} p<0.05 ;{ }^{* *} p<0.01 ;{ }^{\star \star *} p<0.001 ;{ }^{* \star \star} p<0.0001$.

increase in cAMP production of $8.6 \pm 2.6$ - and $6.7 \pm 0.8$-fold change/basal, respectively (Figure 3Aii). There were no significant effects of either FSH24 or dg-eLHt, on cAMP production at this time point (Figure 3Aii). When compared and correlated with PD-PALM data, a trend was observed for 2- minute $30 \mathrm{ng} / \mathrm{ml} \mathrm{eFSH}$ and FSH21/18 promoting dissociation of FSHR homomers into predominantly monomers (Figures 1Aii, iii), suggesting that dissociation of FSHR oligomers into monomers and re-organization of FSHR oligomeric complexes may, at least in part, promote acute cAMP production. 
A

(i)

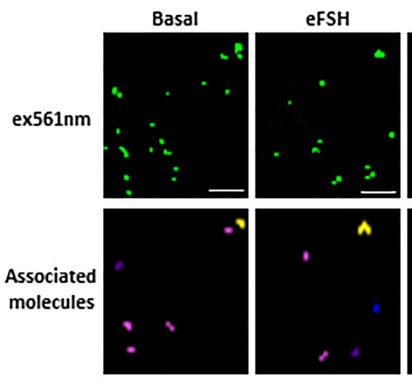

(ii)

B

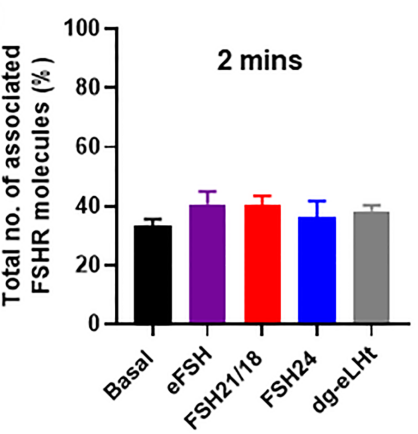

(i)

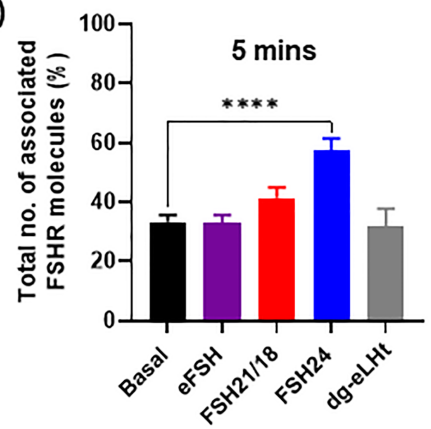

C

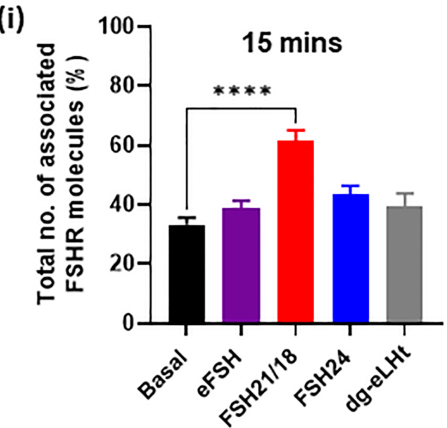

FSH21/18
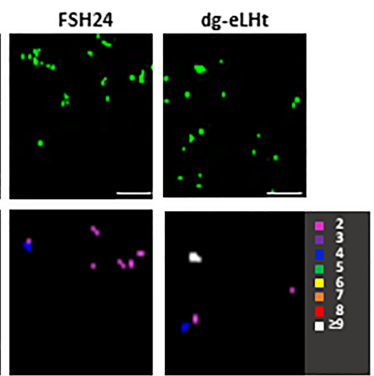

(iii)

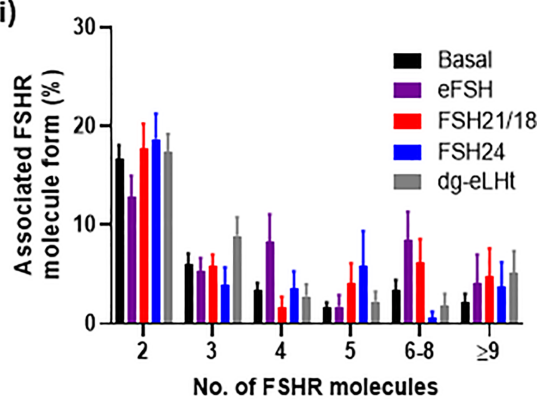

(ii)

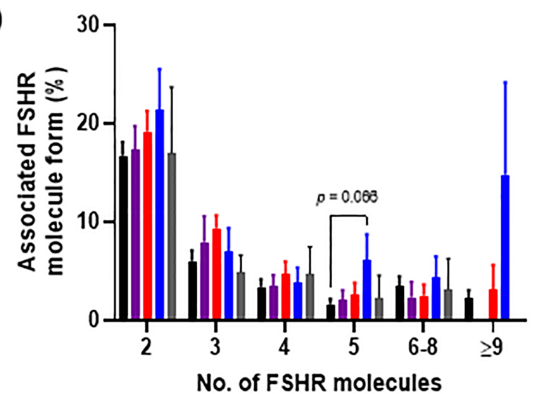

(ii)

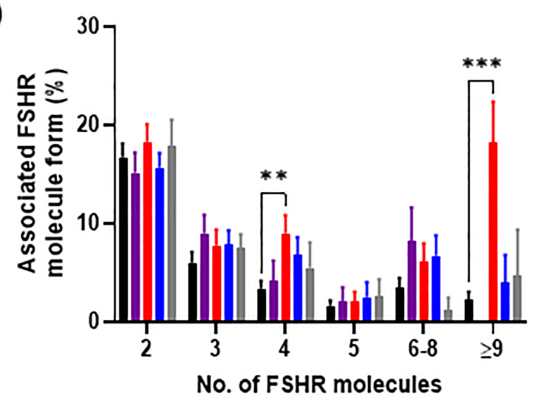

FIGURE 2 | Low FSH ligand concentrations have distinct temporal effects on FSHR association. HEK293 cells expressing HA-tagged FSHR, labelled with CAGE 552HA antibody and treated with $\pm 1 \mathrm{ng} / \mathrm{ml}$ of eFSH, FSH21/18, FSH24 or dg-eLHt for either (A) 2 minutes, (B) 5 minutes or (C) 15 minutes. Cells were fixed and imaged via PD-PALM. (Ai) Representative resolved localized FSHR molecules following 2-minute treatment (top panel) and heat map showing associated FSHR molecules). Images are $2 \mu \mathrm{m}^{2}$ from a $5 \mu \mathrm{m}^{2}$ area. Scale bars, 500nm. (Aii), (Bi) and (Ci) The percentage of the total number of associated FSHR molecules; data analyzed using ordinary one-way ANOVA. (Aiii), (Bii) and (Cii) The percentage of associated FSHR molecule form; 2 (dimer), 3 (trimer), 4 (tetramer), 5 (pentamer), 6-8, $\geq 9$; data analyzed using multiple unpaired t-tests. All data represent mean \pm SEM of 3 independent experiments. ${ }^{\star} p<0.05 ;{ }^{* \star} p<0.01 ;{ }^{\star \star \star} p<0.001 ;{ }^{\star \star \star \star} p<0.0001$.

Moreover, that no change or enhancement of FSHR oligomerization may facilitate low level production of cAMP.

Treatment for 5-minutes with FSH24 significantly increased cAMP (Figure 3Aiii). When compared to observations with PD-
PALM data, a decrease in FSHR association at 5-minutes FSH24 treatment was observed (Figure 1B). This provided further support that FSHR dissociation into monomers may promote cAMP production. Differences in the magnitude of cAMP 
A (i)

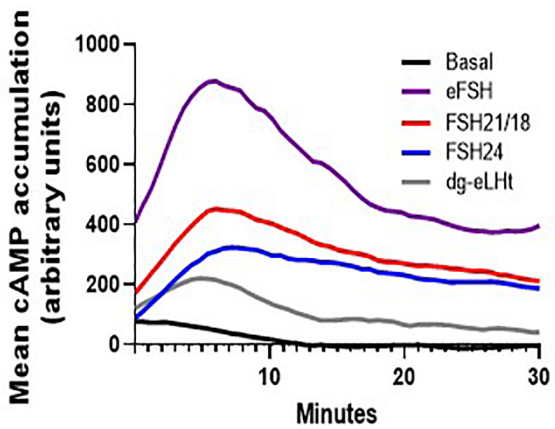

(i)

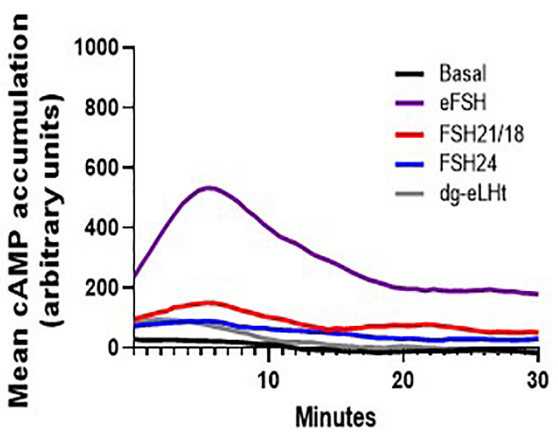

(ii)

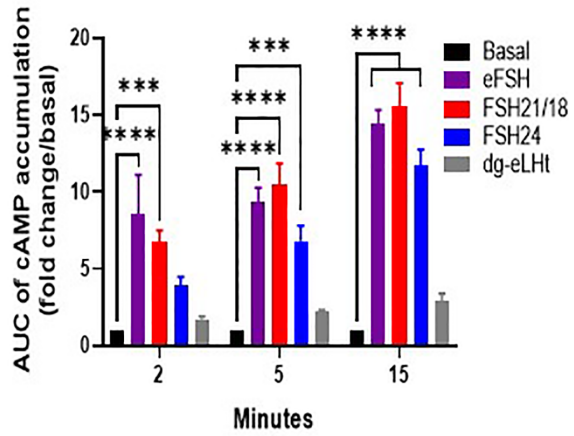

(ii)

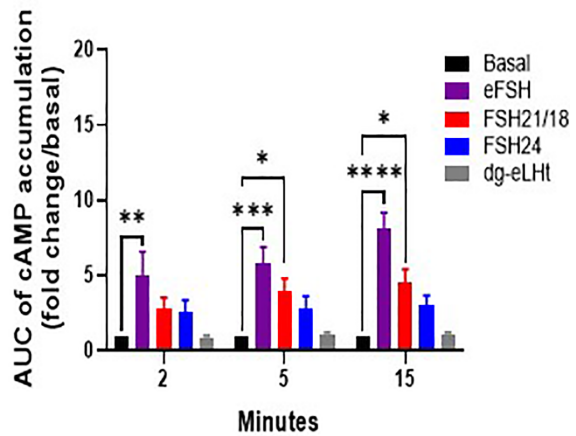

FIGURE 3 | Different concentrations of FSH ligands differentially modulate cAMP production in a temporal manner. HEK293 cells expressing the HA-tagged FSHR

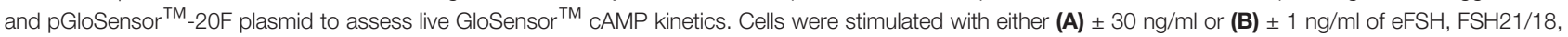
FSH24 or dg-eLHt. (i) Smoothened curve of the mean cAMP accumulation following treatment over 30 minutes (no error bars). (ii) AUC of the mean liganddependent cAMP accumulation at 2-, 5- and 15 minutes. AUC data was baseline subtracted and represented as fold change/basal. Data analyzed using two-way ANOVA. All data represent mean \pm SEM of $3-5$ independent experiments, measured in triplicate. ${ }^{*} p<0.05 ;{ }^{\star \star} p<0.01 ;{ }^{\star \star \star} p<0.001 ;{ }^{\star \star \star \star} p<0.0001$.

accumulation between eFSH, FSH21/18 and FSH24 were observed (Supplementary Figure S1) and may result from the temporal differences in the kinetics of FSHR homomeric complex dissociation and profile of FSHR homomers favored by different FSHR ligands. We observed predominantly eFSHand FSH21/18-dependent pentamer dissociation with acute treatment (Figures 1Aiii, Bii) compared to FSH24-dependent FSHR dimer and trimer dissociation (Figure 1Bii). The dg-eLHt preparation failed to significantly stimulate cAMP production (Figure 3Aii), as compared to PD-PALM data, which showed increased FSHR oligomerization (Figure 1).

A 15-minute stimulation with either eFSH, FSH21/18 or FSH24 continued to significantly increase cAMP production (Figure 3Aii). However, from the maximal cAMP concentration-response curves (Supplementary Figure S1Cii), steady-state levels appeared to have been reached. Interestingly, at this time point, FSHR homomer arrangements predominantly resembled basal conditions in all treatment groups (Figure 1C), suggesting that this receptor configuration may be important in initiating FSHR signal activation, with other mechanisms such as receptor internalization important in maintaining cAMP production thereafter. As anticipated, dg-eLHt was unable to induce significant CAMP production at any time point analyzed (Figure 3Aii). PD-PALM data at the corresponding time point showing preferential re-arrangement of FSHR into higher order oligomers (Figure 1), suggesting that low level cAMP production (and potential $\beta$-arrestin recruitment and subsequent signaling) may be mediated, at least in part, by FSHR oligomer formation.

Next, we determined the effects of lower FSHR ligand concentrations that differentially modulated FSHR homomerization, on cAMP production. As with our previous PD-PALM experiments, we utilized $1 \mathrm{ng} / \mathrm{ml}$ of eFSH, FSH21/18, FSH2 4 or dg-eLHt and measured the mean cAMP accumulated over 30 minutes (Figure 3Bi). An acute, 2-minute treatment with all ligands, except eFSH, showed minimal increases in cAMP production in comparison to basal (Figure 3Bii). When compared to the PD-PALM data (Figure 2), these data correlated with a lack of effect on FSHR oligomerization at 2 minutes, following $1 \mathrm{ng} / \mathrm{ml}$ treatment with any FSHR ligand (Figures 2Aii, iii). 
At 5- and 15 minutes, although we observed an eFSHdependent increase in cAMP production of $5.8 \pm 1.1$ - and $8.1 \pm$ 1.1-fold, respectively (Figure 3Bii), when correlated to the PDPALM data at these time points, no changes in the total percentage of FSHR homomers at the plasma membrane were observed (Figure 2). This suggests that there may be a dosedependent threshold for different FSH glycosylated ligands to modulate FSHR homomerization. Small changes were observed in FSHR homomer subtypes, which may be important for modulating the magnitude of cAMP signaling, however this remains to be demonstrated. In contrast, FSH24 treatment at 5- and 15-minutes had no significant effect on cAMP production (Figure 3Bii). Interestingly, when correlating PD-PALM analysis, an increase in FSHR oligomerization was observed, predominately from enhanced formation of pentamers (Figure 2Bii), which may indicate that low level cAMP production may favor FSHR association. Supporting this observation, we observed increases in the total percentage of FSHR homomers with FSH21/18 treatment at 15 minutes (Figure 2Ci), correlating with low level cAMP production at the same time (Figure 3Bii). As anticipated, no significant changes in cAMP were observed following 2-, 5-, or 15-minute treatment with dg-eLHt (Figure 3Bii).

\section{FSHR Ligands Differentially Modulate Cre-Luciferase Activity}

As the principal pathway activated by FSH/FSHR is Gos/cAMP/ PKA, which is physiologically important for regulating the expression of cre-response genes, including CYP19 essential for estradiol production $(16,19,40-42)$, we went on to determine the effects of FSHR ligands on cre-luciferase reporter gene activity. HEK293 cells transiently expressing the HA-FSHR were co-transfected with cre-luciferase and $R$-luciferase (transfection efficiency control) and treated with $\pm 0-100 \mathrm{ng} / \mathrm{ml}$ of eFSH, FSH21/18, FSH24 and dg-eLHt for 4-6 hours. In line with the GloSensor ${ }^{\mathrm{TM}}$ data, eFSH and $\mathrm{FSH} 21 / 18$ were most potent at activating cre-luciferase for all concentrations $\geq 1 \mathrm{ng} /$ $\mathrm{ml}$ when compared to basal in contrast to FSH24-treated cells (Figure 4A). As anticipated, dg-eLHt was unable to induce any changes in cre-luciferase activity at lower concentrations. However, at the higher concentrations of $30-$ and $100 \mathrm{ng} / \mathrm{ml}$, dg-eLHt appeared to act as a weak agonist at the FSHR (Figure 4A), in corroboration with previous reports (36).

Comparison of $30 \mathrm{ng} / \mathrm{ml}$ treatments with FSHR ligands showed ligand-dependent differences in cre-luciferase activation, with eFSH and FSH21/18 significantly stimulating cre-luciferase activity by $19.3 \pm 2.6$ - and $17.8 \pm 0.7$-fold increase over basal, respectively, in comparison to an $11.8 \pm 0.8$-fold increase for FSH24-treated cells $(p<0.0001)$ (Figure 4B). This reflects similar differences observed between eFSH, FSH21/18 and FSH24 in $30 \mathrm{ng} / \mathrm{ml}$ GloSensor ${ }^{\mathrm{TM}}$ cAMP data (Figure 3Aii). Furthermore, when compared to FSH ligands eliciting changes in FSH homomerization, it suggests that the changes observed in FSHR complexes at the plasma membrane may contribute to modulating the magnitude of cre-responsive gene activation.

Comparison of cre-luciferase responses following $1 \mathrm{ng} / \mathrm{ml}$ treatment with eFSH, FSH21/18, FSH24 and dg-eLHt revealed both eFSH and FSH21/18 induced 7-fold increases in creluciferase activation $(p<0.001)$ (Figure 4B). Additionally, FSH24 induced a comparable increase $(6.1 \pm 0.2$-fold over basal) in cre-luciferase activity. This was interesting as differential regulation of FSHR homomeric forms and cAMP production was observed at this concentration. As predicted, dgeLHt failed to significantly induce any increase in cre-luciferase activity (Figure 4B), further supporting its $\beta$-arrestin biased agonist activity.
A

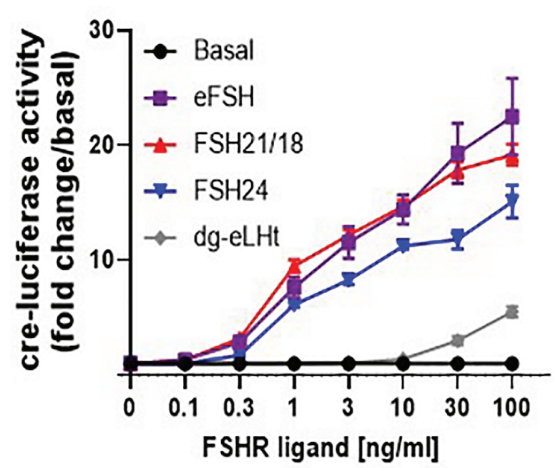

B

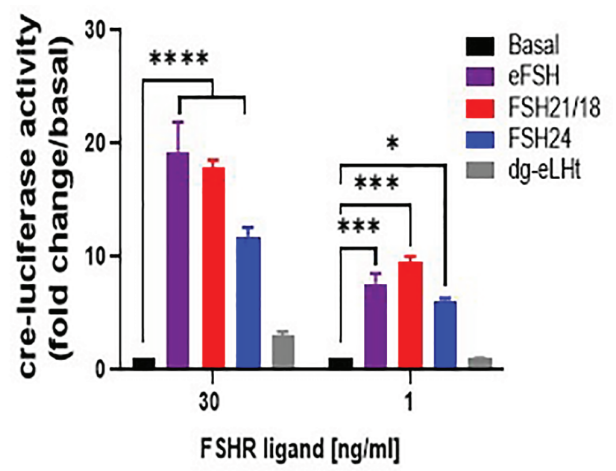

FIGURE 4 | Differential FSH ligands stimulate cre-luciferase activity in a concentration-dependent manner. HEK293 cells were transiently expressing HA-tagged FSHR, cre-luciferase and Renilla luciferase plasmids. Cells were treated in serum-free media for 4-6 hours with increasing concentrations (0-100 ng/ml) of eFSH, FSH21/18, FSH24 or dg-eLHt. (A) concentration-dependent effects of FSH ligands on cre-luciferase activity, represented as fold change/basal. (B) Extrapolation of data from (A) to measure the effect of \pm 30 - and $\pm 1 \mathrm{ng} / \mathrm{ml}$ of eFSH, FSH21/18, FSH24 or dg-eLHt on cre-luciferase activity. Data represented as fold change/basal and analyzed using ordinary two-way ANOVA. All data points were normalized to the internal transfection efficiency control, Renilla-luciferase. Each data point represents mean \pm SEM for 3-5 independent experiments, measured in triplicate. ${ }^{*} p<0.05 ;{ }^{* *} p<0.01 ;{ }^{* * *} p<0.001 ;{ }^{* \star * \star} p<0.0001$. 


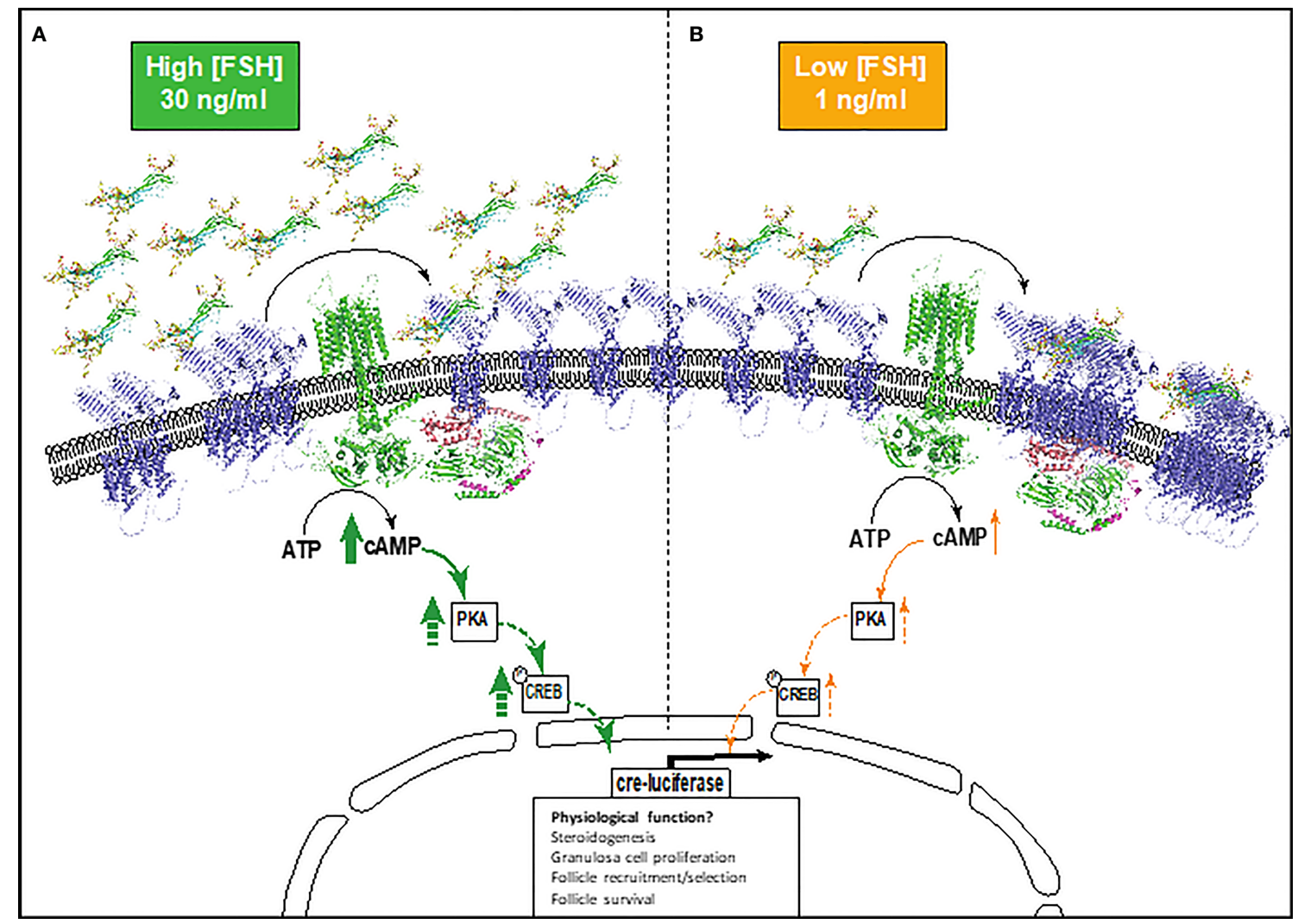

FIGURE 5 | Schematic depicting the proposed model of FSHR monomer/oligomer-dependent modulation of cAMP signaling. (A) Higher physiological concentrations of FSH glycoforms (30 ng/ml), mediates the re-organization of FSHR oligomers into monomers at the plasma membrane, activating the Gos-adenylyl cyclase-cAMP-PKA-CREB pathway and resulting in high cre-responsive gene activation. (B) Lower physiological concentrations of FSH glycoforms (1 ng/ml). FSHR monomer-homomer populations remain quiescent or further associate to promote FSHR oligomerization. This results in lower levels of cAMP and cre-responsive gene activation. This may have implications on granulosa cell functions including steroidogenesis, granulosa cell proliferation, follicle recruitment and selection, and follicle survival. Dashed arrows indicate proteins in the cAMP pathway that were not investigated, but are concordant with published literature. FSH glycoforms are modelled, FSHR is represented by an alpha-fold model (blue) and structural depiction of adenylyl cyclase (green).

\section{DISCUSSION}

FSH glycosylation variants have been previously shown to display differences in the magnitude and specificity of pathways activated $(15,17,19,43)$. Yet how FSHR decodes and propagates such signal diversity and differences in signal amplitude and duration remains unknown. GPCR homomerization is a well-recognized mechanism for modulating functional diversity and specifying signal responses $(44,45)$. Here we propose a mechanism for FSH glycoform-specific temporal and concentration-dependent regulation of FSHR homomerization, which correlates modulation of the amplitude and temporal activation of cAMP signaling (Figure 5).

We have shown that pituitary FSH glycoforms regulate FSHR homomerization in a time- and concentration-dependent manner. At higher physiological concentrations, eFSH and FSH21/18 rapidly dissociated FSHR homomers predominantly into monomers, correlating with significant increases in eFSH and FSH21/18-dependent cAMP production (Figure 5A). Interestingly FSH24 displayed slower temporal kinetics of modulating FSHR homomerization, but dissociated FSHR homomers into predominantly monomers at time points when cAMP production was significantly increased. These data are in concordance with early studies of the related glycoprotein hormone receptor, TSHR, where FRET and co-immunoprecipitation analysis revealed less active dimer and oligomer conformations dissociated into monomers upon TSH stimulation (46). Conversely, at high concentrations of the $\beta$-arrestin biased agonist, dg-eLHt, a rapid increase in FSHR homomerization was observed. For the purpose of this study, this FSHR biased agonist was used as a negative control for cAMP production, and this increase in FSHR oligomerization correlated with a limited ability to activate cAMP, consistent with previous reports (36). These data suggest that defined FSHR monomer, dimer and oligomeric forms may at least in part, modulate the magnitude of cAMP production. This proposition is interesting as FSH glycoforms have been demonstrated to be dynamically regulated during ageing, with modulation in the ratio of FSH21/18 and FSH24 and overall circulating levels of FSH as ovarian ageing proceeds (13), and reported changes in secretion across the menstrual cycle $(13,39)$. It 
is tempting to speculate that modulation of FSH glycoform and concentrations during these physiological processes may modulate FSHR homomer subtype and the dynamic shift between monomer-dimer-oligomer forms. This in turn may specify the amplitude and duration of CAMP signaling, with potential for signal specific physiological outcomes. Such FSH glycoform specific modulation of FSHR complexes and tempering of cAMP production may present ways for therapeutic exploitation in assisted reproduction. However, further research is required to understand the molecular detail and physiological control of this. These data also have implication for other GPCRs reported to homomerize/heteromerize, as ligand concentration is often overlooked and is particularly important for receptors with endogenous ligands that have diurnal/circadian/cyclical changes in secretion.

The glycoprotein hormone receptors have been previously reported to display inherent negative cooperativity, or functional asymmetry (26). This has been described for homomers for many GPCRs $(47,48)$ and has been proposed as a mechanism for mediating more graded responses. It has additionally been suggested that negative cooperativity may play an important role in many biological responses as it can cause marked threshold and ultra-sensitivity, allowing a biological system to filter out small stimuli and respond decisively to suprathreshold stimuli (48). Our observations of FSHR dissociation at high ligand concentrations and FSHR association/no change with low ligand concentrations support this idea, whereby FSHR oligomerization decodes the ligand threshold to regulate signal activation. In a physiological context within the ovary, such regulation may help prevent mass activation of FSHR, and finetune FSHR function during the fluctuations in FSH concentrations that are observed in different phases of folliculogenesis such as follicle recruitment, selection and ovulation $(6,49,50)$.

Differences in binding affinity and the number of FSHR sites occupied by FSH21/18 and FSH24 have previously been reported, with FSH21 displaying a higher binding affinity to FSHR and occupying more FSHR (51, 52). Additionally, competition binding assays have shown that unlabeled eFSH and FSH21/18 was more efficacious at displacing ${ }^{125} \mathrm{I}-\mathrm{FSH} 24$ and ${ }^{125} \mathrm{I}-\mathrm{FSH} 21 / 18$ at lower concentrations than unlabeled hFSH24 (14), supporting the differences in FSHR binding affinities. In the context of this study, it is possible that these reported differences in the binding properties of the FSH glycoforms may have implications for the temporal differences observed in FSHR oligomer re-arrangement and dissociation into monomers observed with eFSH and FSH21/18 versus FSH24. However, future studies are required to determine how FSH glycoformdependent differences in FSHR binding affinity and kinetics may drive changes in FSHR oligomerization.

We utilized the FSHR biased agonist, dg-eLHt, with known preferential $\beta$-arrestin signaling at lower concentrations $(\leq 1 \mathrm{nM})$ and weak cAMP activation (36), and observed concentration dependent modulation of FSHR oligomerization, with enhanced association at high concentrations. For other GPCRs, agonist dependent induction of homomerization has also been observed, including the dopamine D2 receptor homodimers (53). As dgeLHt is a preferentially recruits $\beta$-arrestin, which has well established roles in receptor desensitization and internalization, we can't rule out the induction of FSHR clustering, rather than FSHR oligomerization for initiation of FSHR internalization, particularly at high ligand concentrations. Indeed, interesting next steps will be to explore the effects of FSH glycoforms on the desensitization, internalization and trafficking of FSHR. It will be interesting to unpick how these observed differences in FSHR organization at the plasma membrane may direct FSHR internalization and trafficking, and understand the relationship between canonical Gos coupling and $\beta$-arrestin recruitment and signaling. The association of FSHR and $\beta$-arrestin has long been established, with roles of this molecular scaffold in ERK phosphorylation (18, 54-56), with ligand activation rapidly phosphorylating a cluster of residues within the $\mathrm{C}$ terminus of FSHR to facilitate $\beta$-arrestin recruitment and receptor internalization (57). Interestingly, a recent study has suggesting FSH glycoform-specific differences in the dependency of $\beta$ arrestin for ERK activation (17). With the recently reported roles of ligand-dependent differences in regulatory 'phosphorylation barcodes' for other Class A GPCRs (58), it may be that FSH ligands generate differential phosphorylation barcodes resulting in ligand-specific modulation of FSHR trafficking and signal propagation. Recent reports have suggested that internalization of FSHR is required for initiation of FSH-dependent cAMP production (59), with low molecular weight FSHR agonists reported to differentially modulate FSHR exocytosis (59), which may explain differential profiles for activating cAMP. How FSH glycoforms direct FSHR internalization and trafficking, remains to be determined. However, the use of single molecule imaging and single particle tracking presents exciting opportunities to determine the spatial-temporal regulation of these processes and uncover how/if different FSHR complexes are routed through the endosomal machinery to modulate FSH liganddependent signaling.

FSH glycoforms have been demonstrated to activate additional non-canonical $\mathrm{G}$ protein-signaling including $\mathrm{G} \alpha \mathrm{q}$ and $\mathrm{G} \alpha \mathrm{i}$ linked pathways $(56,60,61)$. It will be interesting to determine if FSHR oligomerization contributes to determining and specifying $G$ protein-coupling within a FSHR oligomeric/ monomer complex. Indeed, observations with LHR/FSHR heteromers support this idea, whereby an increase in heterotetramers was reported to drive the enhanced LH/LHRdependent Goq/11 signaling (32). Interestingly, the formation of FSHR and membrane bound estrogen receptors (GPER) heteromers (FSHR-GPER) have been recently reported (33), with a proposed role in reprogramming FSHR-related death signals into life signals, as a result of high density FSHRs (62) and/or too high cAMP (63). These data support the physiological roles of FSHR homomers and heteromers with potentially distinct biological functions. This may be important for extragonadal FSHR functions where FSH/FSHR has been

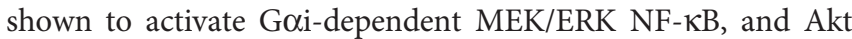
signaling to enhance osteoclast formation (64-66) and Goi- 
dependent induction of uncoupling protein-1 expression for FSH-dependent regulation of adipocytes $(65,67)$. However, this remains to be determined.

This study highlighted that $\sim 70 \%$ of FSHR basally resided as monomers. This is in concordance with previous studies with the gonadotrophin hormone receptors, where the LHR was reported to reside as $\sim 60 \%$ as monomers (34). Earlier studies into the Class A rhodopsin GPCR revealed that the majority of these receptors function as monomers despite their high concentrations within the plasma membrane (68). An important next step is to understand the role of FSHR monomers, and how they regulate the functionality and physiological responses of FSHR.

One of the limitations of this study is the utilization of HEK293 cells to study FSHR oligomerization, with transfected FSHR expression. A primary function of ovarian granulosa cells, in which FSHR are endogenously expressed, is to produce estrogen via aromatase expression. As a steroidogenic cell, the granulosa cell plasma membrane environment is cholesterol rich (69). The local membrane environment is increasingly recognized as an important factor regulating GPCR function (70-72) and GPCR homomer formation (73). To begin to understand the physiological context of this study findings, an important next step is to translate these findings into physiologically relevant cell types.

In conclusion, we have demonstrated that differential FSHR ligands modulate FSHR homomerization in a concentration and time-dependent manner. These data suggest that modulation of FSHR homomerization may provide a mechanism to finetune signal specificity and amplitude. This may be important means to decode the occurring cyclical and age-dependent changes in FSH concentration and glycosylation patterns in both a physiological and pathophysiological context. Moreover, modulation of FSHR homomerization may provide potential novel therapeutic avenues for targeting FSHR to improve IVF outcomes.

\section{REFERENCES}

1. Chu L, Li J, Liu Y, Cheng CH. Gonadotropin Signaling in Zebrafish Ovary and Testis Development: Insights From Gene Knockout Study. Mol Endocrinol (2015) 29(12):1743-58. doi: 10.1210/me.2015-1126

2. Kumar TR, Wang Y, Lu N, Matzuk MM. Follicle Stimulating Hormone is Required for Ovarian Follicle Maturation But Not Male Fertility. Nat Genet (1997) 15(2):201-4. doi: 10.1038/ng0297-201

3. Kumar TR. Fshb Knockout Mouse Model, Two Decades Later and Into the Future. Endocrinology (2018) 159(5):1941-9. doi: 10.1210/en.2018-00072

4. Stilley JAW, Segaloff DL. FSH Actions and Pregnancy: Looking Beyond Ovarian FSH Receptors. Endocrinology (2018) 159(12):4033-42. doi: 10.1210/en.2018-00497

5. Zhang Z, Lau SW, Zhang L, Ge W. Disruption of Zebrafish FollicleStimulating Hormone Receptor (Fshr) But Not Luteinizing Hormone Receptor (Lhcgr) Gene by TALEN Leads to Failed Follicle Activation in Females Followed by Sexual Reversal to Males. Endocrinology (2015) 156 (10):3747-62. doi: 10.1210/en.2015-1039

6. Son WY, Das M, Shalom-Paz E, Holzer H. Mechanisms of Follicle Selection and Development. Minerva Ginecol (2011) 63(2):89-102.

7. Messinis IE, Messini CI, Dafopoulos K. Novel Aspects of the Endocrinology of the Menstrual Cycle. Reprod BioMed Online (2014) 28(6):714-22. doi: 10.1016/j.rbmo.2014.02.003

\section{DATA AVAILABILITY STATEMENT}

The original contributions presented in the study are included in the article/Supplementary Material. Further inquiries can be directed to the corresponding author.

\section{AUTHOR CONTRIBUTIONS}

KJ and GB conceived the study. KJ, GB, and AA supervised the study. UA, EC, and KJ carried out experiments. UA, EC, and KJ analyzed data. $\mathrm{UA}, \mathrm{KJ}, \mathrm{AA}, \mathrm{VB}$, and $\mathrm{GB}$ wrote and revised the manuscript. All authors contributed to the article and approved the submitted version.

\section{FUNDING}

This work was supported by National Institutes of Health (P01AG029531), BBSRC grant (BB/R015961/1 and BB/ R015961/2), and Society for Reproduction and Fertility Covid19 support scheme for PhD Students 2021.

\section{ACKNOWLEDGMENTS}

We thank the Facility for Imaging of Light Microscopy at Imperial College London for technical support in PD-PALM imaging.

\section{SUPPLEMENTARY MATERIAL}

The Supplementary Material for this article can be found online at: https://www.frontiersin.org/articles/10.3389/fendo.2021.765727/ full\#supplementary-material

8. Baker VL, Fujimoto VY, Kettel LM, Adamson GD, Hoehler F, Jones CE, et al. Clinical Efficacy of Highly Purified Urinary FSH Versus Recombinant FSH in Volunteers Undergoing Controlled Ovarian Stimulation for In Vitro Fertilization: A Randomized, Multicenter, Investigator-Blind Trial. Fertil Steril (2009) 91(4):1005-11. doi: 10.1016/j.fertnstert.2008.01.064

9. Cheon KW, Byun HK, Yang KM, Song IO, Choi KH, Yoo KJ. Efficacy of Recombinant Human Follicle-Stimulating Hormone in Improving Oocyte Quality in Assisted Reproductive Techniques. J Reprod Med (2004) 49 (9):733-8.

10. Palagiano A, Nesti E, Pace L. FSH: Urinary and Recombinant. Eur J Obstet Gynecol Reprod Biol (2004) 115(Suppl 1):S30-3. doi: 10.1016/j.ejogrb. 2004.01.023

11. Gleicher N, Kushnir VA, Barad DH. Worldwide Decline of IVF Birth Rates and Its Probable Causes. Hum Reprod Open (2019) 2019(3):hoz017. doi: 10.1093/hropen/hoz017

12. Bousfield GR, Harvey DJ. Follicle-Stimulating Hormone Glycobiology. Endocrinology (2019) 160(6):1515-35. doi: 10.1210/en.2019-00001

13. Bousfield GR, Butnev VY, Rueda-Santos MA, Brown A, Hall AS, Harvey DJ. Macro- and Micro-Heterogeneity in Pituitary and Urinary FollicleStimulating Hormone Glycosylation. J Glycomics Lipidomics (2014) 4:1000124. doi: 10.4172/2153-0637.1000125

14. Bousfield GR, Butnev VY, Hiromasa Y, Harvey DJ, May JV. HypoGlycosylated Human Follicle-Stimulating Hormone (hFSH(21/18)) is Much 
More Active In Vitro Than Fully-Glycosylated hFSH (hFSH(24)). Mol Cell Endocrinol (2014) 382(2):989-97. doi: 10.1016/j.mce.2013.11.008

15. Jiang C, Hou X, Wang C, May JV, Butnev VY, Bousfield GR, et al. Hypoglycosylated hFSH Has Greater Bioactivity Than Fully Glycosylated Recombinant hFSH in Human Granulosa Cells. J Clin Endocrinol Metab (2015) 100(6):E852-60. doi: 10.1210/jc.2015-1317

16. Simon LE, Liu Z, Bousfield GR, Kumar TR, Duncan FE. Recombinant FSH Glycoforms Are Bioactive in Mouse Preantral Ovarian Follicles. Reproduction (2019) 158(6):517-27. doi: 10.1530/REP-19-0392

17. Zariñán T, Butnev VY, Gutiérrez-Sagal R, Maravillas-Montero JL, MartínezLuis I, Mejía-Domínguez NR, et al. In Vitro Impact of FSH Glycosylation Variants on FSH Receptor-Stimulated Signal Transduction and Functional Selectivity. J Endocr Soc (2020) 4(5):bvaa019. doi: 10.1210/jendso/bvaa019

18. Landomiel F, De Pascali F, Raynaud P, Jean-Alphonse F, Yvinec R, Pellissier LP, et al. Biased Signaling and Allosteric Modulation at the FSHR. Front Endocrinol (Lausanne) (2019) 10:148. doi: 10.3389/fendo.2019.00148

19. Wang H, May J, Butnev V, Shuai B, May JV, Bousfield GR, et al. Evaluation of In Vivo Bioactivities of Recombinant Hypo- (FSH). Mol Cell Endocrinol (2016) 437:224-36. doi: 10.1016/j.mce.2016.08.031

20. Hua G, George JW, Clark KL, Jonas KC, Johnson GP, Southekal S, et al. HypoGlycosylated hFSH Drives Ovarian Follicular Development More Efficiently Than Fully-Glycosylated hFSH: Enhanced Transcription and PI3K and MAPK Signaling. Hum Reprod (2021) 36(7):1891-906. doi: 10.1093/humrep/deab135

21. Lai TH, Lin YF, Wu FC, Tsai YH. Follicle-Stimulating Hormone-Induced Galphah/phospholipase C-Delta1 Signaling Mediating a Noncapacitative Ca2 + Influx Through T-Type Ca2+ Channels in Rat Sertoli Cells. Endocrinology (2008) 149(3):1031-7. doi: 10.1210/en.2007-1244

22. Sleno R, Hébert TE. The Dynamics of GPCR Oligomerization and Their Functional Consequences. Int Rev Cell Mol Biol (2018) 338:141-71. doi: 10.1016/bs.ircmb.2018.02.005

23. Wang W, Qiao Y, Li Z. New Insights Into Modes of GPCR Activation. Trends Pharmacol Sci (2018) 39(4):367-86. doi: 10.1016/j.tips.2018.01.001

24. Breitwieser GE. G Protein-Coupled Receptor Oligomerization: Implications for G Protein Activation and Cell Signaling. Circ Res (2004) 94(1):17-27. doi: 10.1161/01.RES.0000110420.68526.19

25. Rios CD, Jordan BA, Gomes I, Devi LA. G-Protein-Coupled Receptor Dimerization: Modulation of Receptor Function. Pharmacol Ther (2001) 92 (2-3):71-87. doi: 10.1016/S0163-7258(01)00160-7

26. Urizar E, Montanelli L, Loy T, Bonomi M, Swillens S, Gales C, et al. Glycoprotein Hormone Receptors: Link Between Receptor Homodimerization and Negative Cooperativity. EMBO J (2005) 24(11):1954-64. doi: 10.1038/ sj.emboj.7600686

27. Thomas RM, Nechamen CA, Mazurkiewicz JE, Muda M, Palmer S, Dias JA. Follice-Stimulating Hormone Receptor Forms Oligomers and Shows Evidence of Carboxyl-Terminal Proteolytic Processing. Endocrinology (2007) 148 (5):1987-95. doi: 10.1210/en.2006-1672

28. Guan R, Wu X, Feng X, Zhang M, Hébert TE, Segaloff DL. Structural Determinants Underlying Constitutive Dimerization of Unoccupied Human Follitropin Receptors. Cell Signal (2010) 22(2):247-56. doi: 10.1016/ j.cellsig.2009.09.023

29. Jiang X, Fischer D, Chen X, McKenna SD, Liu H, Sriraman V, et al. Evidence for Follicle-Stimulating Hormone Receptor as a Functional Trimer. J Biol Chem (2014) 289(20):14273-82. doi: 10.1074/jbc.M114.549592

30. Mazurkiewicz JE, Herrick-Davis K, Barroso M, Ulloa-Aguirre A, LindauShepard B, Thomas RM, et al. Single-Molecule Analyses of Fully Functional Fluorescent Protein-Tagged Follitropin Receptor Reveal Homodimerization and Specific Heterodimerization With Lutropin Receptor. Biol Reprod (2015) 92(4):100. doi: 10.1095/biolreprod.114.125781

31. Feng X, Zhang M, Guan R, Segaloff DL. Heterodimerization Between the Lutropin and Follitropin Receptors is Associated With an Attenuation of Hormone-Dependent Signaling. Endocrinology (2013) 154(10):3925-30. doi: 10.1210/en.2013-1407

32. Jonas KC, Chen S, Virta M, Mora J, Franks S, Huhtaniemi I, et al. Temporal Reprogramming of Calcium Signalling via Crosstalk of Gonadotrophin Receptors That Associate as Functionally Asymmetric Heteromers. Sci Rep (2018) 8(1):2239. doi: 10.1038/s41598-018-20722-5

33. Casarini L, Lazzaretti C, Paradiso E, Limoncella S, Riccetti L, Sperduti S, et al. Membrane Estrogen Receptor (GPER) and Follicle-Stimulating
Hormone Receptor (FSHR) Heteromeric Complexes Promote Human Ovarian Follicle Survival. iScience (2020) 23(12):101812. doi: 10.1016/j.isci. 2020.101812

34. Jonas KC, Fanelli F, Huhtaniemi IT, Hanyaloglu AC. Single Molecule Analysis of Functionally Asymmetric G Protein-Coupled Receptor (GPCR) Oligomers Reveals Diverse Spatial and Structural Assemblies. J Biol Chem (2015) 290 (7):3875-92. doi: 10.1074/jbc.M114.622498

35. Jonas KC, Hanyaloglu AC. Analysis of Spatial Assembly of GPCRs Using Photoactivatable Dyes and Localization Microscopy. Methods Mol Biol (2019) 1947:337-48. doi: 10.1007/978-1-4939-9121-1_19

36. Wehbi V, Tranchant T, Durand G, Musnier A, Decourtye J, Piketty V, et al. Partially Deglycosylated Equine LH Preferentially Activates Beta-ArrestinDependent Signaling at the Follicle-Stimulating Hormone Receptor. Mol Endocrinol (2010) 24(3):561-73. doi: 10.1210/me.2009-0347

37. Butnev VY, Singh V, Nguyen VT, Bousfield GR. Truncated Equine LH Beta and Asparagine(56)-Deglycosylated Equine LH Alpha Combine to Produce a Potent FSH Antagonist. J Endocrinol (2002) 172(3):545-55. doi: 10.1677/ joe.0.1720545

38. Herrick-Davis K, Grinde E, Lindsley T, Teitler M, Mancia F, Cowan A, et al. Native Serotonin 5-HT2C Receptors Are Expressed as Homodimers on the Apical Surface of Choroid Plexus Epithelial Cells. Mol Pharmacol (2015) 87 (4):660-73. doi: $10.1124 /$ mol.114.096636

39. Wide L, Eriksson K. Low-Glycosylated Forms of Both FSH and LH Play Major Roles in the Natural Ovarian Stimulation. Ups J Med Sci (2018) 123(2):100-8. doi: 10.1080/03009734.2018.1467983

40. Parakh TN, Hernandez JA, Grammer JC, Weck J, Hunzicker-Dunn M, Zeleznik AJ, et al. Follicle-Stimulating Hormone/cAMP Regulation of Aromatase Gene Expression Requires Beta-Catenin. Proc Natl Acad Sci USA (2006) 103(33):12435-40. doi: 10.1073/pnas.0603006103

41. Stocco C. Aromatase Expression in the Ovary: Hormonal and Molecular Regulation. Steroids (2008) 73(5):473-87. doi: 10.1016/j.steroids.2008.01.017

42. Reichert LE, Dattatreyamurty B. The Follicle-Stimulating Hormone (FSH) Receptor in Testis: Interaction With FSH, Mechanism of Signal Transduction, and Properties of the Purified Receptor. Biol Reprod (1989) 40(1):13-26. doi: 10.1095/biolreprod40.1.13

43. Bousfield GR, May JV, Davis JS, Dias JA, Kumar TR. In Vivo and In Vitro Impact of Carbohydrate Variation on Human Follicle-Stimulating Hormone Function. Front Endocrinol (Lausanne) (2018) 9:216. doi: 10.3389/ fendo.2018.00216

44. Agwuegbo UC, Jonas KC. Molecular and Functional Insights Into Gonadotropin Hormone Receptor Dimerization and Oligomerization. Minerva Ginecol (2018) 70(5):539-48. doi: 10.23736/S0026-4784.18.04287-9

45. Casarini L, Crépieux P. Molecular Mechanisms of Action of FSH. Front Endocrinol (Lausanne) (2019) 10:305. doi: 10.3389/fendo.2019.00305

46. Latif R, Graves P, Davies TF. Ligand-Dependent Inhibition of Oligomerization at the Human Thyrotropin Receptor. J Biol Chem (2002) 277(47):45059-67. doi: 10.1074/jbc.M206693200

47. Rivero-Müller A, Jonas KC, Hanyaloglu AC, Huhtaniemi I. Di/ oligomerization of GPCRs-Mechanisms and Functional Significance. Prog Mol Biol Transl Sci (2013) 117:163-85. doi: 10.1016/B978-0-12-3869319.00007-6

48. Ha SH, Ferrell JE. Thresholds and Ultrasensitivity From Negative Cooperativity. Science (2016) 352(6288):990-3. doi: 10.1126/science.aad5937

49. Driancourt MA. Regulation of Ovarian Follicular Dynamics in Farm Animals. Implications for Manipulation of Reproduction. Theriogenology (2001) 55 (6):1211-39. doi: 10.1016/S0093-691X(01)00479-4

50. Gougeon A. Human Ovarian Follicular Development: From Activation of Resting Follicles to Preovulatory Maturation. Ann Endocrinol (Paris) (2010) 71(3):132-43. doi: 10.1016/j.ando.2010.02.021

51. Butnev VY, May JV, Shuai B, Tran P, White WK, Brown A, et al. Production, Purification, and Characterization of Recombinant hFSH Glycoforms for Functional Studies. Mol Cell Endocrinol (2015) 405:42-51. doi: 10.1016/ j.mce.2015.01.026

52. Davis JS, Kumar TR, May JV, Bousfield GR. Naturally Occurring FollicleStimulating Hormone Glycosylation Variants. J Glycomics Lipidomics (2014) 4 (1):e117. doi: 10.4172/2153-0637.1000e117

53. Tabor A, Weisenburger S, Banerjee A, Purkayastha N, Kaindl JM, Hübner H, et al. Visualization and Ligand-Induced Modulation of Dopamine Receptor 
Dimerization at the Single Molecule Level. Sci Rep (2016) 6:33233. doi: $10.1038 /$ srep33233

54. De Pascali F, Reiter E. $\beta$-Arrestins and Biased Signaling in Gonadotropin Receptors. Minerva Ginecol (2018) 70(5):525-38. doi: 10.23736/S00264784.18.04272-7

55. Lefkowitz RJ, Shenoy SK. Transduction of Receptor Signals by Beta-Arrestins. Science (2005) 308(5721):512-7. doi: 10.1126/science.1109237

56. Gloaguen P, Crépieux P, Heitzler D, Poupon A, Reiter E. Mapping the Follicle-Stimulating Hormone-Induced Signaling Networks. Front Endocrinol (Lausanne) (2011) 2:45. doi: 10.3389/fendo.2011.00045

57. Kara E, Crépieux P, Gauthier C, Martinat N, Piketty V, Guillou F, et al. A Phosphorylation Cluster of Five Serine and Threonine Residues in the CTerminus of the Follicle-Stimulating Hormone Receptor is Important for Desensitization But Not for Beta-Arrestin-Mediated ERK Activation. Mol Endocrinol (2006) 20(11):3014-26. doi: 10.1210/me.2006-0098

58. Dwivedi-Agnihotri H, Chaturvedi M, Baidya M, Stepniewski TM, Pandey S, Maharana J, et al. Distinct Phosphorylation Sites in a Prototypical GPCR Differently Orchestrate $\beta$-Arrestin Interaction, Trafficking, and Signaling. Sci $A d v$ (2020) 6(37):eabb8368. doi: 10.1126/sciadv.abb8368

59. Sposini S, De Pascali F, Richardson R, Sayers NS, Perrais D, Yu HN, et al. Pharmacological Programming of Endosomal Signaling Activated by Small Molecule Ligands of the Follicle Stimulating Hormone Receptor. Front Pharmacol (2020) 11:593492. doi: 10.3389/fphar.2020.593492

60. Stilley JA, Guan R, Santillan DA, Mitchell BF, Lamping KG, Segaloff DL. Differential Regulation of Human and Mouse Myometrial Contractile Activity by FSH as a Function of FSH Receptor Density. Biol Reprod (2016) 95(2):36. doi: 10.1095/biolreprod.116.141648

61. Crépieux P, Marion S, Martinat N, Fafeur V, Vern YL, Kerboeuf D, et al. The ERK-Dependent Signalling Is Stage-Specifically Modulated by FSH, During Primary Sertoli Cell Maturation. Oncogene (2001) 20(34):4696-709. doi: 10.1038/sj.onc. 1204632

62. Casarini L, Reiter E, Simoni M. $\beta$-Arrestins Regulate Gonadotropin ReceptorMediated Cell Proliferation and Apoptosis by Controlling Different FSHR or LHCGR Intracellular Signaling in the Hgl5 Cell Line. Mol Cell Endocrinol (2016) 437:11-21. doi: 10.1016/j.mce.2016.08.005

63. Aharoni D, Dantes A, Oren M, Amsterdam A. cAMP-Mediated Signals as Determinants for Apoptosis in Primary Granulosa Cells. Exp Cell Res (1995) 218(1):271-82. doi: 10.1006/excr.1995.1156

64. Sun L, Peng Y, Sharrow AC, Iqbal J, Zhang Z, Papachristou DJ, et al. FSH Directly Regulates Bone Mass. Cell (2006) 125(2):247-60. doi: 10.1016/ j.cell.2006.01.051

65. Liu P, Ji Y, Yuen T, Rendina-Ruedy E, DeMambro VE, Dhawan S, et al. Blocking FSH Induces Thermogenic Adipose Tissue and Reduces Body Fat. Nature (2017) 546(7656):107-12. doi: 10.1038/nature22342
66. Zhu LL, Blair H, Cao J, Yuen T, Latif R, Guo L, et al. Blocking Antibody to the $\beta$-Subunit of FSH Prevents Bone Loss by Inhibiting Bone Resorption and Stimulating Bone Synthesis. Proc Natl Acad Sci USA (2012) 109(36):14574-9. doi: 10.1073/pnas.1212806109

67. Liu XM, Chan HC, Ding GL, Cai J, Song Y, Wang TT, et al. FSH Regulates Fat Accumulation and Redistribution in Aging Through the $\mathrm{G \alpha i} / \mathrm{Ca}(2+) / \mathrm{CREB}$ Pathway. Aging Cell (2015) 14(3):409-20. doi: 10.1111/acel.12331

68. Park PS, Filipek S, Wells JW, Palczewski K. Oligomerization of G ProteinCoupled Receptors: Past, Present, and Future. Biochemistry (2004) 43 (50):15643-56. doi: 10.1021/bi047907k

69. Lange Y, Schmit VM, Schreiber JR. Localization and Movement of Newly Synthesized Cholesterol in Rat Ovarian Granulosa Cells. Endocrinology (1988) 123(1):81-6. doi: 10.1210/endo-123-1-81

70. Periole X, Huber T, Marrink SJ, Sakmar TP. G Protein-Coupled Receptors Self-Assemble in Dynamics Simulations of Model Bilayers. J Am Chem Soc (2007) 129(33):10126-32. doi: 10.1021/ja0706246

71. Koldsø H, Sansom MS. Organization and Dynamics of Receptor Proteins in a Plasma Membrane. J Am Chem Soc (2015) 137(46):14694-704. doi: 10.1021/ jacs.5b08048

72. Guixà-González R, Javanainen $\mathrm{M}$, Gómez-Soler $\mathrm{M}$, Cordobilla $\mathrm{B}$, Domingo JC, Sanz F, et al. Membrane Omega-3 Fatty Acids Modulate the Oligomerisation Kinetics of Adenosine A2A and Dopamine D2 Receptors. Sci Rep (2016) 6:19839. doi: 10.1038/srep19839

73. Prasanna X, Sengupta D, Chattopadhyay A. Cholesterol-Dependent Conformational Plasticity in GPCR Dimers. Sci Rep (2016) 6:31858. doi: $10.1038 /$ srep31858

Conflict of Interest: The authors declare that the research was conducted in the absence of any commercial or financial relationships that could be construed as a potential conflict of interest.

Publisher's Note: All claims expressed in this article are solely those of the authors and do not necessarily represent those of their affiliated organizations, or those of the publisher, the editors and the reviewers. Any product that may be evaluated in this article, or claim that may be made by its manufacturer, is not guaranteed or endorsed by the publisher.

Copyright (®) 2021 Agwuegbo, Colley, Albert, Butnev, Bousfield and Jonas. This is an open-access article distributed under the terms of the Creative Commons Attribution License (CC BY). The use, distribution or reproduction in other forums is permitted, provided the original author(s) and the copyright owner(s) are credited and that the original publication in this journal is cited, in accordance with accepted academic practice. No use, distribution or reproduction is permitted which does not comply with these terms. 